\title{
Reelin Glycoprotein
}

Structure, Biology and Roles in Health and Disease 
S. Hossein Fatemi

\section{Reelin Glycoprotein}

Structure, Biology and Roles in Health and Disease

睹 Springer 


\title{
Editor
}

\author{
S. Hossein Fatemi, M.D., Ph.D. \\ Professor of Psychiatry \\ Adjunct Professor of Pharmacology and Neuroscience \\ University of Minnesota \\ Medical School \\ Minneapolis, MN \\ USA \\ fatem002@umn.edu
}

\author{
ISBN: 978-0-387-76760-4 \\ e-ISBN: 978-0-387-76761-1 \\ DOI: $10.1007 / 978-0-387-76761-1$
}

Library of Congress Control Number: 2008920271

(C) 2008 Springer Science+Business Media, LLC

All rights reserved. This work may not be translated or copied in whole or in part without the written permission of the publisher (Springer Science+Business Media, LLC, 233 Spring Street, New York, NY 10013, USA), except for brief excerpts in connection with reviews or scholarly analysis. Use in connection with any form of information storage and retrieval, electronic adaptation, computer software, or by similar or dissimilar methodology now known or hereafter developed is forbidden.

The use in this publication of trade names, trademarks, service marks, and similar terms, even if they are not identified as such, is not to be taken as an expression of opinion as to whether or not they are subject to proprietary rights.

While the advice and information in this book are believed to be true and accurate at the date of going to press, neither the authors nor the editors nor the publisher can accept any legal responsibility for any errors or omissions that may be made. The publisher makes no warranty, express or implied, with respect to the material contained herein.

Printed on acid-free paper

987654321

springer.com 
To my father, S. Mehdi Fatemi, and to my family, S. Ali Fatemi, M.D., Naheed Fatemi, Parvin Fatemi, S. Mohammad Fatemi, Neelufaar Fatemi, Maryam Jalali-Mousavi, and last but not least, my mother, Fatemeh Parsa Moghaddam, whose love and support have enabled me to complete this work. 


\section{Foreword}

By inviting me to contribute a short foreword to this superb collective work on the biology of Reelin, Dr Fatemi gives me a nice opportunity for some reminiscence and speculation. The first reeler mutation appeared in 1948 in Edinburgh, and reeler mice were for many years the only genetic model of cortical malformation. The model became popular in the mid sixties and seventies, thanks to the vision of Sidman and his colleagues, especially Caviness and Rakic. However, in the following years, reeler fell a bit out of fashion because the gene was not characterized. Only a few groups, such as those of Mikoshiba and myself, persisted in studying reeler mice. This changed dramatically when D'Arcangelo and Curran cloned the Reelin gene in 1995. The next few years saw rapid progress with the identification of the adaptor Dab1 by Howell and Cooper in 1997, and of reelin receptors by Herz in 1999. Since 1999, as is often the case after a golden period, progress has been slower. Still, we have witnessed very significant progress that is nicely covered in this book. We understand more about the biochemistry and structure of Reelin, and most probably a full structure of the native protein will be defined in the coming years. Reelin has been studied during brain development in several species, and this resulted in a somewhat clearer view of its importance during brain evolution. Similar comparative studies of Dab1 and receptors would be needed, however, and I doubt that they will be done soon. We know a lot about Reelin expression in the embryonic and adult brain, even though the basic mechanisms that control Reelin synthesis and secretion, particularly by Cajal-Retzius cells, need to be defined better. The proximal steps in the signal elicited by Reelin have basically been worked out, and interactions between proximal signaling by Reelin and other important pathways are being elucidated at increasing pace. In addition, several papers appear regularly that describe expression of Reelin where it is not really expected, generating new hypotheses on what are probably several different functions of a large protein. Whether these various functions use the canonical Reelin signaling pathway or additional pathways that remain to be identified is another theme of interest for the next few years. Last but not least, a large body of evidence has accumulated that hints at a role of Reelin in psychiatric disorders.

Notwithstanding this vast body of exciting data, however, we are still in the dark about some key issues, among which I would mention two that appear particularly 
important - at least to me. First and foremost, even though Reelin receptors and signaling components have been identified, we still do not know what Reelin does to immature neurons. The concept that Reelin provides a stop signal has been useful but is reaching its operational limit. Presumably, we all agree that Reelin somehow instructs neurons to arrest migration and take position in early architectonic patterns (cortical plate, Purkinje cell layer, etc. ...). However, how this happens remains unknown. Does Reelin modulate expression of adhesion molecules on the surface of neurons, radial glia, or both, as I always imagined? Alternatively, does Reelin signaling impact on the cytoskeleton, thereby "freezing" the architecture of end-migration neurons? Does Reelin signaling recruit or hijack other signaling pathways such as Notch, as recently proposed by the Rakic group? Second, what is the function of Reelin after maturation and in the mature brain. What does Reelin do when it is secreted by cortical interneurons? Related to this are clearly the questions about the actions of Reelin in learning and behavior, and in psychiatric diseases. To address this, we need to inactivate Reelin and/or its partners in neurons after normal maturation, using, for example, floxed alleles and Cre-ER technology. As far as I know, these tools are only being developed.

As this superb book outlines, the Reelin field is at a crossroad. Following a rapid initial phase, with cloning of Reelin, identification of Dab1 and receptors, each of which owed quite a lot to serendipity, progress has been much slower and difficult. Whether future breakthrough will also occur unexpectedly (maybe the next knockout...) or will result from more rational approaches, is anybody's guess. But one thing is more predictable, namely that this timely book will prove very useful and will end up on the bookshelf of all investigators with an interest in the Reelin puzzle. We should all be grateful to Dr Fatemi and his staff for editing it so carefully.

Andre M. Goffinet, MD, PhD Brussels, Belgium 


\section{Preface}

Reelin glycoprotein is a major secretory protein with important roles in embryogenesis and during adult life. Reelin gene mutations or deficiency of the protein product cause abnormal cortical development and Reelin signaling impairment in brain. Since the first discovery of the reelin mutant mouse in 1951 by Falconer, and later discovery of the gene for Reelin in 1995, there has been an explosion of new knowledge about this important molecule. As of this writing, a search of public library of medicine cites over 665 published papers on Reelin.

Thus, it became apparent that a book dealing with this topic and presenting contributions from an international panel of experts would be timely and necessary. In the following twenty eight chapters, various authors will present up-to-date discussions of the state of the knowledge on various aspects of Reelin such as reelin gene, its receptors, downstream effector molecules in Reelin signaling cascade, chemistry and structure of Reelin, comparative anatomy of reelin, presence of Reelin in various body tissues, Reelin mutations, and abnormalities of Reelin production in neuropsychiatric disorders and cancer.

It is hoped that this book serves as a foundation for analysis of this emerging novel protein for all interested neuroscientists and clinicians.

S. Hossein Fatemi, M.D., Ph.D.

Minneapolis, Minnesota 


\section{Acknowledgments}

Many have helped to make the publication of this book possible. I am especially indebted to Ms. Teri Jane Reutiman, who faithfully reviewed all chapters for accuracy and worked as a liaison between the editor and the authors of the chapters, and Mr. Timothy D. Folsom for help with various aspects of editing this book. I am grateful to Ms. Laurie Iversen for clerical assistance. I am also grateful to the publishers and authors who have generously given approval for reproduction of tables and figures, as well as to Ms. Kathleen Lyons, Ms. Dana Andreachi, and Mr. Brian Halm at Springer Science + Business Media, and Ms. Padmasani Srimadhan at SPi Publisher Services for an excellent job in publishing this book.

S. Hossein Fatemi 


\section{Contents}

Contributors

xvii

1 The Reelin Gene and Its Functions in Brain Development Cheng-Chiu Huang and Gabriella D'Arcangelo

2 Apolipoprotein E Receptor 2 and Very-Low-Density

Lipoprotein Receptor: An Overview

Hans H. Bock and Joachim Herz

3 Chemistry of Reelin.

Yves Jossin

4 The C-Terminal Region of Reelin: Structure and Function

Mitsuharu Hattori

5 Crystal Structure of Reelin Repeats

Junichi Takagi

6 Comparative Anatomy and Evolutionary Roles of Reelin

Gundela Meyer

7 Reelin/Dab1 Signaling in the Developing Cerebral Cortex

Eric C. Olson and Christopher A. Walsh

8 Ultrastructural Localization of Reelin

Rosalinda C. Roberts and Emma Perez-Costas

9 Reelin and Cyclin-Dependent Kinase 5

Toshio Ohshima

10 Reelin and the Cerebellum

Robert F. Hevner 
11 Reelin and Radial Glial Cells

Eckart Förster, Shanting Zhao, and Michael Frotscher

12 Reelin and Cognition.

Shenfeng Qiu and Edwin John Weeber

13 Protein Kinases and Signaling Pathways

that Are Activated by Reelin

Jonathan A. Cooper, Nathaniel S. Allen, and Libing Feng

14 The Relationship of Oxytocin and Reelin in the Brain

George D. Pappas and C. Sue Carter

15 Reelin and Thyroid Hormone

Manuel Álvarez-Dolado

16 A Tale of Two Genes: Reelin and BDNF

Thomas Ringstedt

17 Reelin, Liver, and Lymphatics.

Brigitte Samama and Nelly Boehm

18 Reelin and Cajal-Retzius Cells

Jean-Marc Mienville

19 Reelin and Odontogenesis.

Françoise Bleicher, Henry Magloire, Marie-Lise Couble, and Jean-Christophe Maurin

20 Homozygous and Heterozygous Reeler Mouse Mutants

Patricia Tueting, Graziano Pinna, and Erminio Costa

21 Reelin and Lissencephaly

Elena Parrini and Renzo Guerrini

22 The Role of Reelin in Etiology and Treatment of Psychiatric Disorders

S. Hossein Fatemi, Teri J. Reutiman, and Timothy D. Folsom

23 Reelin Downregulation as a Prospective Treatment Target

for GABAergic Dysfunction in Schizophrenia..

Erminio Costa, Ying Chen, Erbo Dong, Dennis R. Grayson, Alessandro Guidotti, and Marin Veldic 
24 Epigenetic Modulation of Reelin Function in Schizophrenia and Bipolar Disorder

Hamid Mostafavi Abdolmaleky, Cassandra L. Smith, Jin-Rong Zhou, and Sam Thiagalingam

25 Reelin Gene Polymorphisms in Autistic Disorder

Carla Lintas and Antonio Maria Persico

26 Alzheimer's Disease and Reelin

Arancha Botella-López and Javier Sáez-Valero

27 Reelin and Stroke Kunlin Jin

28 Reelin and Pancreatic Cancer

Kimberly Walter and Michael Goggins

Index 


\section{Contributors}

Hamid Mostafavi Abdolmaleky, MD

Senior Research Associate, Biomedical Engineering Department, Boston University, Boston; Laboratory of Nutrition and Metabolism at BIDMC, Department of Surgery, Harvard Medical School, Boston; Departments of Medicine (Genetics Program), Genetics \& Genomics, and Pathology \& Laboratory Medicine, Boston University School of Medicine, Boston, MA USA and

Assistant Professor of Psychiatry, Department of Psychiatry, Tehran Psychiatric Institute and Mental Health Research Center, Iran University of Medical Sciences, Tehran, Iran

Nathaniel S. Allen, BS

Graduate Student, Division of Basic Sciences, Fred Hutchinson Cancer Research Center, Seattle, WA USA

Manuel Álvarez-Dolado, $\mathrm{PhD}$

Ramón y Cajal Researcher, Team Leader of Cellular Regeneration Laboratory, Centro de Investigación Príncipe Felipe (CIPF), Valencia, Spain

Françoise Bleicher, PhD, MS

Professor of Biochemistry, University of Lyon 1, Odontoblast and Dental Tissue Regeneration Team, Faculty of Odontology, Lyon, France

Nelly Boehm, MD, PhD

Professor of Histology, Institut d'Histologie, Faculté de Médecine, Université Louis Pasteur, Strasbourg, France; _INSERM U666, Strasbourg, France; Hôpitaux Universitaires de Strasbourg, France

Hans H. Bock, MD

Research Fellow, Zentrum für Neurowissenschaften, Universität Freiburg, Freiburg, Germany 
Arancha Botella-López, BS

Research Assistant, Instituto de Neurociencias de Alicante, Universidad Miguel Hernández-CSIC, Sant Joan d'Alacant, Spain, and Centro de Investigación

Biomédica en Red sobre Enfermedades Neurodegenerativas (CIBERNED), Spain

C. Sue Carter, $\mathrm{PhD}$

Professor of Psychiatry, Department of Psychiatry, Co-Director of The Brain Body Center, the Psychiatric Institute, College of Medicine, University of Illinois at Chicago, Chicago, IL USA

Ying Chen, MD

Research Specialist, the Psychiatric Institute, Department of Psychiatry, College of Medicine, University of Illinois at Chicago, Chicago, IL USA

Jonathan A. Cooper, PhD

Member, Division of Basic Sciences, Fred Hutchinson Cancer Research Center, Seattle, WA USA

Erminio Costa, MD

Distinguished Professor of Biochemistry and Psychiatry, Director of the Psychiatric Institute, Department of Psychiatry, College of Medicine, University of Illinois at Chicago, Chicago, IL USA

Marie-Lise Couble, MS

Research Assistant, University of Lyon 1, Odontoblast and Dental Tissue

Regeneration Team, Faculty of Odontology, Lyon, France

Gabriella D'Arcangelo, PhD

Associate Professor, Department of Cell Biology and Neuroscience, Rutgers, the State University of New Jersey, Piscataway, NJ USA

Erbo Dong, $\mathrm{PhD}$

Research Assistant Professor of Psychiatry, the Psychiatric Institute, Department of Psychiatry, College of Medicine, University of Illinois at Chicago, Chicago, IL USA

S. Hossein Fatemi, MD, PhD

Professor of Psychiatry, Adjunct Professor of Pharmacology and Neuroscience, Departments of Psychiatry, Pharmacology, and Neuroscience, University of Minnesota Medical School, Minneapolis, MN USA

Libing Feng, PhD

Postdoctoral Fellow, Division of Basic Sciences, Fred Hutchinson Cancer Research Center, Seattle, WA USA 
Timothy D. Folsom, MS

Research Assistant, Department of Psychiatry, University of Minnesota Medical School, Minneapolis, MN USA

Eckart Förster, PhD

Professor of Anatomy, Institut für Anatomie I, Zelluläre Neurobiologie, Universität Hamburg, Hamburg, Germany

Michael Frotscher, MD

Professor of Anatomy, Institut für Anatomie und Zellbiologie, Abteilung für Neuroanatomie, Universität Freiburg, Freiburg, Germany

Michael Goggins, MD

Director of the Pancreatic Cancer Early Detection Laboratory, Associate Professor of Pathology, Medicine, and Oncology, Departments of Pathology, Medicine, and Oncology, The Sol Goldman Pancreatic Research Center, The Johns Hopkins Medical Institutions, Baltimore, MD USA

Dennis R. Grayson, PhD

Professor of Molecular Psychiatry, the Psychiatric Institute, Department of

Psychiatry, College of Medicine, University of Illinois at Chicago, Chicago, IL USA

Renzo Guerrini, MD

Professor of Child Neurology and Psychiatry, Director - Child Neurology Unit, Pediatric Hospital A. Meyer-University of Firenze, Pediatric Neurology Unit and Laboratories, Children's Hospital A. Meyer-University of Florence, Florence, Italy

Alessandro Guidotti, MD

Professor of Psychiatry, Scientific Director, the Psychiatric Institute,

Department of Psychiatry, College of Medicine, University of Illinois at Chicago, Chicago, IL USA

Mitsuharu Hattori, $\mathrm{PhD}$

Associate Professor, Department of Biomedical Science, Graduate School of Pharmaceutical Sciences, Nagoya City University, Nagoya, Aichi, Japan

Joachim Herz, MD

Professor of Biophysics and Molecular Genetics, Thomas O. Hicks Family

Distinguished Chair in Alzheimer's Disease Research, Department of Molecular Genetics, University of Texas Southwestern Medical Center, Dallas, TX USA and

Zentrum für Neurowissenschaften, Universität Freiburg, Freiburg, Germany

Robert F. Hevner, MD, PhD

Associate Professor and Neuropathologist, Department of Pathology/

Neuropathology, University of Washington, Seattle, WA USA 
Cheng-Chiu Huang, MS

Graduate Program in Developmental Biology, Baylor College of Medicine, Houston, TX USA; Graduate Student, PhD Program in Developmental Biology, Baylor College of Medicine, Houston, TX USA

Kunlin Jin, MD, PhD

Associate Research Professor, Buck Institute for Age Research, Novato, CA USA

Yves Jossin, PhD

Postdoctoral Fellow, Université Catholique de Louvain, Faculté de Médecine, Developmental Neurobiology Unit, Brussels, Belgium and

Research Associate, Fred Hutchinson Cancer Research Center, Division of Basic Sciences, Seattle, WA USA

Carla Lintas, $\mathrm{PhD}$

Research Scientist, Laboratory of Molecular Psychiatry and Neurogenetics, University "Campus Bio-Medico," and Department of Experimental Neurosciences, I.R.C.C.S. "Fondazione Santa Lucia," Rome, Italy

Henry Magloire, PhD, DDS

Professor of Basic Sciences in Oral Biology, University of Lyon 1, Odontoblast and Dental Tissue Regeneration Team, Faculty of Odontology, Lyon, France

Jean-Christophe Maurin, PhD, DDS

Assistant Professor of Operative Dentistry, Interfaces Biomatériaux/Tissus Hôtes, Faculty of Odontology of Reims, Reims, France

Gundela Meyer, MD, PhD

Professor of Anatomy, Departamento de Anatomía, Facultad de Medicina, Universidad de La Laguna, La Laguna, Spain

Jean-Marc Mienville, $\mathrm{PhD}$

Professor, Laboratoire de Physiologie Cellulaire et Moléculaire, CNRS - UMR 6548, Université de Nice-Sophia Antipolis, Parc Valrose, Nice, France

Toshio Ohshima, MD, PhD

Professor and Head of Laboratory for Molecular Brain Science, Department of Life Science and Medical Bio-Science, Waseda University, Sinjuku, Tokyo, Japan

Eric C. Olson, PhD

Assistant Professor of Neuroscience and Physiology, Department of Neuroscience and Physiology, SUNY Upstate Medical University, Syracuse, NY USA 
George D. Pappas, PhD

Professor of Psychiatry, Anatomy and Cell Biology, Department of Psychiatry, the Psychiatric Institute, College of Medicine, University of Illinois at Chicago, Chicago, IL USA

Elena Parrini, $\mathrm{PhD}$

Neurogenetics Lab, Pediatric Neurology Unit and Laboratories, Children's Hospital A. Meyer-University of Florence, Florence, Italy

\section{Emma Perez-Costas, $\mathrm{PhD}$}

Assistant Professor, Department of Psychiatry and Behavioral Neurobiology, University of Alabama at Birmingham, Birmingham, AL USA

\section{Antonio Maria Persico, MD}

Associate Professor of Physiology, Head of Laboratory of Molecular Psychiatry and Neurogenetics, University "Campus Bio-Medico," and Department of Experimental Neurosciences, I.R.C.C.S. "Fondazione Santa Lucia," Rome, Italy

Graziano Pinna, $\mathrm{PhD}$

Research Assistant Professor of Psychiatry (Neuroendocrinology), the Psychiatric Institute, Department of Psychiatry, University of Illinois at Chicago, Chicago, IL USA

Shenfeng Qiu, PhD

Postdoctoral Fellow, Department of Molecular Physiology and Biophysics, Vanderbilt University Medical Center, Nashville, TN USA

Teri J. Reutiman, BA

Junior Scientist, Department of Psychiatry, University of Minnesota Medical School, Minneapolis, MN USA

Thomas Ringstedt, PhD

Assistant Professor of Developmental Neurobiology, Neonatal Unit, Karolinska Institutet, Astrid Lindgren Children's Hospital, Stockholm, Sweden

Rosalinda C. Roberts, PhD

Professor of Psychiatry, Kathy Ireland Endowed Chair for Psychiatric Research, Department of Psychiatry and Behavioral Neurobiology, University of Alabama at Birmingham, Birmingham, AL USA

Javier Sáez-Valero, $\mathrm{PhD}$

Assistant Professor of Biochemistry, Instituto de Neurociencias de Alicante, Universidad Miguel Hernández-CSIC, Sant Joan d'Alacant, Spain and Centro de Investigación Biomédica en Red sobre Enfermedades Neurodegenerativas (CIBERNED), Spain 
Brigitte Samama, $\mathrm{PhD}$

Associate Professor of Histology, Institut d'Histologie, Faculté de Médecine, Université Louis Pasteur, Strasbourg, France; I_NSERM U666, Strasbourg, France; Hôpitaux Universitaires de Strasbourg, France

Cassandra L. Smith, $\mathrm{PhD}$

Professor of Biomedical Engineering, Pharmacology and Experimental

Therapeutics, School of Medicine, Director, Molecular Biotechnology

Research Laboratory, Biomedical Engineering Department, Boston University, Boston, MA USA

Junichi Takagi, PhD

Professor, Institute for Protein Research, Laboratory of Protein Synthesis and Expression, Osaka University, Suita, Osaka, Japan

Sam Thiagalingam, $\mathrm{PhD}$

Associate Professor of Medicine, Genetics/Genomics, Pathology and Laboratory Medicine, Departments of Medicine (Genetics Program), Genetics and Genomics, and Pathology and Laboratory Medicine, Boston University School of Medicine, Boston, MA USA

Patricia Tueting, PhD

Research Assistant Professor of Psychiatry (Neuroscience), the Psychiatric Institute, Department of Psychiatry, College of Medicine, University of Illinois at Chicago, Chicago, IL USA

Marin Veldic, MD

Research Assistant Professor of Psychiatry, the Psychiatric Institute, Department of Psychiatry, College of Medicine, University of Illinois at Chicago, Chicago, IL USA

Christopher A. Walsh, MD, PhD

Bullard Professor of Neurology at Harvard Medical School, Chief of the Division of Genetics at Boston Children's hospital, Director of the Harvard-MIT M.D.-

Ph.D. Program, and Investigator of the Howard Hughes Medical Institute at Beth

Israel Deaconess Medical Center, Howard Hughes Medical Institute, BIDMC, Division of Genetics, Boston Children's Hospital, Harvard Medical School, Boston, MA USA

Kimberly Walter, BS

Graduate Student in the Pathobiology and Disease Mechanisms Program at Johns Hopkins University, Department of Pathology, The Sol Goldman Pancreatic Research Center, The Johns Hopkins Medical Institutions, Baltimore, MD USA 
Edwin John Weeber, PhD

Associate Professor, Department of Molecular Pharmacology and Physiology,

University of South Florida, Tampa, FL USA

Shanting Zhao, MD

Senior Scientist, Institut für Anatomie und Zellbiologie, Abteilung für

Neuroanatomie, Universität Freiburg, Freiburg, Germany

Jin-Rong Zhou, PhD

Assistant Professor of Surgery and Nutrition, Laboratory of Nutrition and Metabolism at BIDMC, Department of Surgery, Harvard Medical School, Boston, MA USA 